\title{
A pilot study to determine the normal haematological indices for young Malawian adults in Blantyre, Malawi
}

\section{MR Chisale 1 , P Kumwenda ${ }^{2}$, M Ngwira ${ }^{2}$ B M'baya $^{3}$, BI Chosamata, V Mwapasa $^{5}$}

1. Laboratory Section, Mzuzu Central Hospital, Mzuzu, Malawi

2. Department of Biomedical Sciences, Mzuzu University, Mzuzu, Malawi

3. Malawi Blood Transfusion Service, Blantyre, Malawi

4. Department of Pathology and Laboratory Sciences, College of

Medicine, University of Malawi, Blantyre Malawi

5. Department of Public Health, College of Medicine, University of

Malawi, Blantyre, Malawi

Correspondence to: Master R.O. Chisale

E-mail: masterchisale@gmail.com

\section{Abstract}

\section{Background}

Reference ranges for haematological and other laboratory tests in most African countries are based on populations in Europe and America and, because of environmental and genetic factors, these may not accurately reflect the normal reference ranges in African populations.

\section{Aim}

To determine the distribution of haematological parameters in healthy individuals residing in Blantyre, Malawi. We also examined the effect of sociodemographic and nutritional factors on the haematological variables.

\section{Methods}

We conducted a proof-of-concept cross-sectional study, involving 105 healthy blood donors at Malawi Blood Transfusion Service in Blantyre. Eligible participants were HIV-negative males and females, aged 19 to 35 years, who did not have any evidence of acute or chronic illness, or bloodborne infection. We performed the haematological tests at the MalawiLiverpool Wellcome Trust laboratory in Blantyre, and the screening tests at Malawi Blood Transfusion Service laboratories.

\section{Results}

Out of 170 consenting healthy volunteers, haematological results were available for 105 participants. The proportions of results which were below the lower limit of the manufacturer's reference ranges were $35.2 \%(37 / 105)$ for haemoglobin, $15.2 \%$ (16/105) for neutrophils, $23.8 \%$ (25/105) for eosinophils, and $88.6 \%$ (93/105) for basophils. The proportions of results that were above the upper limit of the manufacturer's reference ranges were 9.5\% (10/105) for platelets and $12.4 \%(13 / 105)$ for monocytes. We also observed that the mean leucocyte and basophil counts were significantly higher in males than females $(\mathrm{p}=$ 0.042 and $p=0.015$, respectively). There were no statistically significant differences in haematological results observed among different ethnic, age, and body mass index groups.

\section{Conclusions}

Over half of otherwise healthy study participants had at least one abnormal haematological result, using previously established foreign standards. More detailed studies are needed to establish locally relevant normal ranges for different age groups and other demographic characteristics of the Malawian population. This will lead to accurate interpretation of laboratory results.

\section{Introduction}

In most African countries, including Malawi, normal reference ranges for laboratory variables used in clinical trial and clinical care are obtained from European and North American populations. ${ }^{1}$ However, there are marked differences in values of haematological variables between normal African populations and those of North America and Europe. ${ }^{2}$ For example, healthy African populations are reported to have lower haemoglobin $(\mathrm{Hb})$ levels, red blood cell (RBC) counts, haematocrits (HCT), mean corpuscular volumes (MCV), and platelet and neutrophil counts, and higher monocyte and eosinophil levels than their Western counterparts. ${ }^{3}$ These differences present challenges in interpreting laboratory results from African countries based on reference ranges developed from other populations. Furthermore, there are variations in the variables among different African ethnic groups. ${ }^{4,5}$

Several factors may account for differences in haematological variables. These include genetics, nutritional status, age, and environmental factors. Of all factors, genetic characteristics of populations are believed to account for the majority of the variations in haematological variables. ${ }^{6,7}$ For example, haematological values for Caucasian adults have been found to be higher than for adults of both African ${ }^{8}$ and AfroCaribbean $^{9-11}$ origin. Even within Africa, there are marked variations in haematological variables among various healthy subpopulations. A previous study in western Kenya found that $\mathrm{Hb}$ and $\mathrm{MCV}$ values for healthy young adults and adolescents were higher than those reported from another study in the highlands west of the Kenyan Rift Valley, ${ }^{2}$ but slightly lower than those derived from Ethiopia, Kampala in Uganda, and Mbeya in Tanzania. ${ }^{12-14}$

Within specific subpopulations, age accounts for most of the variation among haematological variables in healthy individuals. Previous studies in Uganda and other countries in Africa ${ }^{8,12,15,16}$ found significant differences in $\mathrm{Hb}, \mathrm{HCT}$, and $\mathrm{RBC}$ values between adolescent and adult males. Although age is an important determinant of haematological values within populations, significant differences still remain among different populations within the same age groups. For example, healthy under-five children from Africa have lower values of haematological variables than children of the same age from Western populations. ${ }^{12-14,17}$ It has, therefore, been recommended that each population must establish its own "normal" reference values for use in clinical assessments. ${ }^{18}$

Although several studies in Africa have attempted to establish normal haematological reference ranges for their local populations, key limitations have often been identified. For example, one study in Mozambique used finger prick instead of venous samples ${ }^{17}$; another study in Kenya did not screen the participants for malaria, hepatitis, and syphilis ${ }^{8}$; while other studies did not consider the body mass indexes (BMI) of study participants. These factors could have affected values of haematological variables; therefore, the utility of reference ranges from these studies may be limited. To date there have been few attempts (most notably in paediatrics) to define normal haematological indices for Malawians, despite the significant number of clinical trials conducted in the country. Thus, we conducted a study to determine the distribution (median and range) of haematological parameters in healthy, HIV-negative young adults in Blantyre, Malawi. We also assessed the effect of sociodemographic and nutritional factors on these haematological parameters.

\section{Materials and methods Study design and setting}

From mid-July to mid-September 2013, we conducted a 
cross-sectional study involving healthy blood donors at the static site of the Malawi Blood Transfusion Service (MBTS) in Blantyre in southern Malawi. As one of Malawi's major commercial cities, Blantyre is home to people originating from many districts in Malawi.

\section{Study population}

The targeted population consisted of young males and females who were registered as new or repeat blood donors at the MBTS. To be eligible for the study, the blood donors had to be aged between 19 and 35 years, residents of Blantyre District, and give consent to participate in the study. Participants who met any of the following criteria were excluded from the study: those with detectable blood-borne infections such as malaria, HIV, syphilis, or hepatitis; females who were menstruating, pregnant, or lactating; those with any evidence of acute or chronic illness at the time of the study or in the preceding 3 months; those taking medications for any medical condition; those who smoked tobacco or consumed alcohol or had a current or past history of using illicit drugs; those who had experienced significant blood loss, from any cause (for example, surgery or trauma) within the preceding three months; those who had donated blood within the preceding three months; or those who had received a blood transfusion within the preceding 12 months.

\section{Sample size}

The National Committee for Clinical Laboratory Services (NCCL) recommends that studies to establish normal reference ranges should have a minimum sample size of 120 subjects for each demographic subset of study participants. ${ }^{19}$ According to this guideline, at least 240 participants (120 males and 120 females) should have been enrolled into the study. Budgetary constraints, however, resulted in a target of 60 males and 60 females.

\section{Data collection procedures}

Ethical approval for the study was obtained from the University of Malawi's College of Medicine Research and Ethics Committee (COMREC) prior to data collection. Before seeking informed consent we provided a study leaflet to all potential blood donors in order to provide an overview of the study objectives, rationale, procedures, risks, and benefits. Thereafter, MBTS staff conducted a routine medical assessment of the blood donors as per their normal procedures and completed standard forms, which captured some of the items in the eligibility criteria. The completed standard forms were reviewed by the principal investigator to exclude those who did not meet some of the study eligibility criteria. Informed consent was sought from those who met all of the eligibility criteria captured during the standard MBTS assessment.

After consent was granted, study team members administered a study-specific questionnaire to assess whether the consenting individuals satisfied the eligibility criteria additional to those that were routinely captured on the standard MBTS forms. Once a consenting participant had satisfied the eligibility criteria, his or her venous blood was collected into a blood collection bag, as per the standard MBTS blood collection procedure. Thereafter, blood left over from the tube connecting the cannula and the blood bag was immediately transferred into blood collection tubes as follows: $3 \mathrm{~mL}$ into a K2 EDTA tube, $5 \mathrm{~mL}$ into another K2 EDTA tube and $3 \mathrm{~mL}$ into a plain vacutainer tube. The 5 $\mathrm{mL}$ of blood collected into the plain and K2 EDTA tube was used for blood screening tests at MBTS, while the $3 \mathrm{~mL}$ tube was used for haematological tests at the Malawi-Liverpool Wellcome Trust (MLW) laboratory in Blantyre.

\section{Laboratory analyses \\ Haematological analysis}

Approximately $1 \mathrm{~mL}$ of the whole blood in the $3 \mathrm{~mL}$ EDTA tube was used to perform absolute white blood cell (WBC) counts (including differential counts), RBC counts (along with associated variables, such as $\mathrm{Hb}, \mathrm{HCT}, \mathrm{MCV}$, and mean corpuscular haemoglobin $[\mathrm{MCH}])$, and platelets counts. A Beckman Coulter HmX haematology analyzer was used to perform these analyses. Clotted or turbid blood samples, and those with signs of severe lipaemia were not analyzed because they are known produce unreliable and inaccurate haematological variables.

\section{Screening tests}

As part of the standard procedure at MBTS, $5 \mathrm{~mL}$ of blood collected in plain and K2 EDTA tubes were screened for HIV, syphilis, and hepatitis using enzyme immunoassays (EIAs) or haemagglutination (HA) and particle agglutination (PA) assays, as appropriate. In addition, thick blood slides were prepared, stained with Field's stain and examined for malaria parasites. The study used results from the standard screening tests to exclude all participants who did not meet the laboratory-based eligibility criteria.

\section{Quality control protocols}

As part of the standard procedure at MBTS, $5 \mathrm{~mL}$ of blood coThe haematology tests were performed at MLW laboratory, a reputable and international-standard laboratory, which has been performing laboratory assays for a large number of clinical trials conducted at Queen Elizabeth Central Hospital for over 20 years. The laboratory has active External Quality Assessment (EQA) registration with internationally reputable quality control agencies such as the United Kingdom National External Quality Assessment Service (UK NEQAS, for haematology assays). In addition to the EQA system, the laboratory performs internal quality assurance tests by running known standards at the start of each day and weekly, as well as running reproducibility tests before testing samples.

\section{Data management and analysis}

Sociodemographic and clinical data were collected in a case report forms (CRFs) while laboratory data were recorded in excel spreadsheets. The lead investigators cross checked each CRF and spreadsheet to detect any transcription errors. Thereafter, data were double-entered into a Microsoft Access database, which was imported into Statistical Package for Social Science (SPSS) software (version 16.0) for analyses. Data analyses focused on performing descriptive statistics. We also generated a measures of central tendency (median) and spread (range). We compared our data against reference limits from the machine used, which are based on a European population. The severity of "abnormalities" was classified using the Division of AIDS (DAIDS) criteria. ${ }^{20}$ We used the t-test to compare the distribution of continuous variables between gender, age, ethnic and BMI categories. A two-sided p-value of 0.05 was considered significant.

\section{Results \\ Characteristics of study participants}

A total of 170 blood donors were screened for their eligibility to participate in the study. Fifty $(29.4 \%)$ of the donors were excluded based on the defined exclusion criteria, which aimed http://dx.doi.org/10.4314/mmj.v27i3.5 
at eliminating participants with known medical conditions, or who were involved in any incident or activity which would likely affect their haematological variables. Blood samples were collected from the remaining 120 individuals. Of the 120 samples collected, two were excluded because of of clotting and 13 were excluded because of positive screening tests results for blood-borne infections such as malaria, HIV, viral hepatitis, and syphilis. The remaining 105 participants' samples were included in the study for analysis. Table 1 shows the baseline characteristics of the recruited study participants. The study participants were young adults (median age $=25$ years), and over one-third of them were students. The participants were predominantly male and over one-third belonged to the Lomwe tribe. The BMIs of most study participants was within the normal range, as per World Health Organization (WHO) criteria.

Table 1: Demographic characteristics of the participants

\begin{tabular}{|c|c|c|c|}
\hline Characteristic & & \multicolumn{2}{|c|}{$\mathrm{N}=105$} \\
\hline \multicolumn{4}{|l|}{$\operatorname{Sex}(\%)$} \\
\hline & Male & 96 & (91.4) \\
\hline & Female & 9 & (8.6) \\
\hline \multicolumn{4}{|l|}{ Ethnic group (\%) } \\
\hline & Yao & 18 & (17.1) \\
\hline & Ngoni & 18 & $(17.1)$ \\
\hline & Lomwe & 38 & $(36.2)$ \\
\hline & Others & 31 & (29.5) \\
\hline \multicolumn{4}{|l|}{ Occupation (\%) } \\
\hline & Manual work & 34 & $(32.4)$ \\
\hline & Non-manual work & 26 & $(24.8)$ \\
\hline & Not working & 7 & (6.7) \\
\hline & Students & 31 & $(36.2)$ \\
\hline Age in years, median & & & 25 \\
\hline (range) & & & $(19.3-34.7)$ \\
\hline Height $(\mathrm{m})$, median & & & 1.66 \\
\hline (range) & & & $(1.50-1.85)$ \\
\hline Weight $(\mathrm{kg})$, median & & & 60 \\
\hline (range) & & & $(45-80)$ \\
\hline BMI $\left(\mathrm{kg} / \mathrm{m}^{2}\right)$, median & & & 22.0 \\
\hline (range) & & & $(17.8-29.7)$ \\
\hline
\end{tabular}

\section{Haematological parameters for enrolled study participants}

Almost all variables generated some values that were outside the lower and upper reference limits provided by the manufacturers of the machine used (Table 2). The proportions of out-of-range values ranged from $1.0 \%$ $(1 / 105)$ to $88.6 \%(93 / 105)$. The majority of out-of-range results for $\mathrm{Hb}$ concentration and absolute counts of neutrophils, eosinophils and basophils were below the lower reference limits. In contrast, the majority of out-of-range results for platelet and monocyte counts were above the upper reference limits.

\section{Classification of severity of out-of-range haematological results}

Classification of out-of-range values for six of the haematological variables was done using the DAIDS grading criteria. ${ }^{20}$ As shown in Table 3 , based on these criteria, up to $55.0 \%(58 / 105)$ of our otherwise healthy study participants would have erroneously been considered to have at least one grade 1-4 haematological adverse event (AE). Some of the abnormal values for $\mathrm{Hb}$ concentration, neutrophil count, and platelets were classified as grade 3 (severe) or 4 (potentially life-threatening).

\section{Discussion}

In clinical practice, haematologic tests are necessary and important in diagnosing and monitoring diseases. In clinical trials, the tests are used to assess eligibility of subjects to participate in trials and for monitoring safety of medical interventions over time. In this study, we found that values of haematological variables for a significant number of otherwise normal Malawians had values outside the haematology machine reference ranges, suggesting abnormalities. According to the DAIDS criteria, most of the abnormal values were graded as mild (grade 1) or moderate (grade 2) in severity. However, some subjects had thrombocytopenia and neutropenia that was graded as severe or potentially lifethreatening. Based on our haematological reference ranges, at least one-third of our healthy study participants would have been reported as having AEs.

The specific abnormal haematological variables found in this study are consistent with previous studies conducted in other African countries. A similar study done in western $\mathrm{Kenya}^{17}$ also found eosinophilia, neutropenia, and leucopoenia in otherwise normal individuals. In the Kenyan study, abnormal values were also graded as severe and potentially life-threatening. Likewise, studies in Uganda, Tanzania and Ethiopia ${ }^{8,13,14}$ found lower $\mathrm{Hb}$ concentrations among African subjects compared to North American and European reference ranges and controls. In addition, other investigations conducted in Uganda, Kenya, Ghana, and South Africa ${ }^{2,14,21,22}$ showed higher eosinophil counts in African subjects compared with their North American counterparts. Furthermore, research done in Uganda, Ethiopia, Zambia, and Nigeria ${ }^{3,5,13,14}$ revealed high prevalences of thrombocytopenia in African populations, based on reference ranges from Western populations.

Table 2: Analysis of haematological parameters for enrolled study participants $(N=105)$ and comparison with reference ranges for the haematological analyzer

\begin{tabular}{|c|c|c|c|c|}
\hline Parameter (units) & Median (ranges) & $\begin{array}{l}\text { Normal reference } \\
\text { limits for the } \\
\text { analyzer }\end{array}$ & $\begin{array}{l}\text { Number and (\%) of } \\
\text { values below the } \\
\text { lower reference for } \\
\text { the analyzer }\end{array}$ & $\begin{array}{l}\text { Number and (\%) of } \\
\text { values above the } \\
\text { upper reference for } \\
\text { the analyzer }\end{array}$ \\
\hline WBC $\left(\times 10^{9} / L\right)$ & $4.3(2.2-8.7)$ & $2.8-7.2$ & $6(5.7)$ & $2(1.9)$ \\
\hline $\operatorname{RBC}\left(\times 10^{12} / \mathrm{L}\right)$ & $5.15(4.02-6.55)$ & $4.32-5.66$ & $8(7.6)$ & $15(14.3)$ \\
\hline $\mathrm{Hb}(\mathrm{g} / \mathrm{dL})$ & $14.3(8.5-17.2)$ & $13.6-16.7$ & $37(35.2)$ & $2(1.9)$ \\
\hline HCT (\%) & $43.2(29.4-51.8)$ & $39.0-50.0$ & $19(18.1)$ & $3(2.9)$ \\
\hline $\operatorname{MCV}(\mathrm{fL})$ & $85.5(54.1-100.9)$ & $82.0-98.0$ & $30(28.6)$ & $4(3.8)$ \\
\hline $\mathrm{MCH}(\mathrm{pg})$ & $28.2(15.6-34.6)$ & $24.2-33.1$ & $11(10.5)$ & $2(1.9)$ \\
\hline $\mathrm{MCHC}(\mathrm{g} / \mathrm{dL})$ & $32.8(28.9-35.5)$ & $31.6-34.9$ & $7(6.7)$ & $1(1.0)$ \\
\hline Platelets ( $\left.\times 10^{9} / \mathrm{L}\right)$ & $195(23-351)$ & $115-290$ & $8(7.6)$ & $10(9.5)$ \\
\hline $\begin{array}{l}\text { Absolute neutrophil } \\
\text { count }\left(\times 10^{9} / \text { L) }\right.\end{array}$ & $2.1(0.7-4.5)$ & $0.90-4.20$ & $16(15.2)$ & $2(1.9)$ \\
\hline $\begin{array}{l}\text { Absolute lymphocyte } \\
\text { count }\left(\times 10^{9} / \text { L) }\right.\end{array}$ & $1.7(1.00-3.50)$ & $1.00-3.20$ & $0(0.0)$ & $1(1.0)$ \\
\hline $\begin{array}{l}\text { Absolute monocyte } \\
\text { count }\left(\times 10^{9} / L\right)\end{array}$ & $0.40(0.02-1.70)$ & $0.15-0.58$ & $2(1.9)$ & $13(12.4)$ \\
\hline $\begin{array}{l}\text { Absolute eosinophil } \\
\text { count }\left(\times 10^{\prime} / \mathrm{L}\right)\end{array}$ & $0.10(0.00-3.20)$ & $0.02-0.79$ & $25(23.8)$ & $8(7.6)$ \\
\hline $\begin{array}{l}\text { Absolute basophil } \\
\text { count }\left(\times 10^{9} / \mathrm{L}\right)\end{array}$ & $0.00(0.00-0.20)$ & $0.02-0.10$ & $93(88.6)$ & $1(1.0)$ \\
\hline
\end{tabular}


Table 3: Frequencyand severity of abnormal values for study participants $(N=105)$ using the DAIDS classification criteria ${ }^{20}$

\begin{tabular}{|c|c|c|c|c|c|c|c|c|c|c|}
\hline \multirow[t]{3}{*}{ Parameter } & \multicolumn{2}{|c|}{$\begin{array}{l}\text { Abnormal } \\
\text { proportion }\end{array}$} & \multicolumn{8}{|c|}{ Severity grade using the DAIDS criteria } \\
\hline & \multirow[b]{2}{*}{$\mathrm{n}$} & \multirow[b]{2}{*}{$\%$} & \multicolumn{2}{|c|}{ Grade 1} & \multicolumn{2}{|c|}{ Grade 2} & \multicolumn{2}{|c|}{ Grade 3} & \multicolumn{2}{|c|}{ Grade 4} \\
\hline & & & $\mathrm{n}$ & $\%$ & $\mathrm{n}$ & $\%$ & $\mathrm{n}$ & $\%$ & $\mathrm{~N}$ & $\%$ \\
\hline Leucopenia & 21 & 20.0 & 18 & 85.7 & 3 & 14.3 & 0 & 0 & 0 & 0 \\
\hline Anaemia & 62 & 59.0 & 48 & 77.4 & 12 & 19.4 & 2 & 3.2 & 0 & 0 \\
\hline Thrombocytopenia & 18 & 17.1 & 7 & 38.9 & 6 & 33.3 & 4 & 22.2 & 1 & 5.6 \\
\hline Neutropenia & 18 & 17.1 & 24 & 48.0 & 22 & 44.0 & 4 & 8.0 & 0 & 0 \\
\hline Lymphopenia & 1 & 0.95 & 0 & 0 & 0 & 0 & 0 & 0 & 0 & 0 \\
\hline Eosinophilia & 9 & 8.6 & 7 & 77.8 & 2 & 22.2 & 0 & 0 & 0 & 0 \\
\hline
\end{tabular}

Leucopenia (x 10 19 cells $/ \mathrm{L}$ ): Grade $1=2.5-3.5$, Grade $2=1.5-2.4$, Grade $3=1.0-1.4$, Grade $4<1.0$ Anaemia $(\mathrm{g} / \mathrm{dL})$ : Grade $1=12.5-14.5$, Grade $2=10.5-12.4$, Grade $3=8.5-10.4$, Grade $4<8.5$

Thrombocytopenia $\left(\times 10^{9}\right.$ cells $\left./ \mathrm{L}\right)$ : Grade $1=125-140$, Grade $2=100-124$, Grade $3=25-99$, Grade $4<25$

Neutropenia $\left(\mathrm{x} 10^{9}\right.$ cells $\left./ \mathrm{L}\right)$ : Grade $1=1.5-2.0$, Grade $2=1.0-1.5$, Grade $3=0.5-0.9$, Grade $4<0.5$

Lymphopenia $\left(x 10^{9}\right.$ cells $\left./ \mathrm{L}\right)$ : Grade $1=0.8-0.1-0$, Grade $2=0.5-0.7$, Grade $3=0.3-0.5$, Grade $4<0.3$

Eosinophilia ( $10^{9}$ cells/L): Grade $1=0.7-1.5$, Grade $2>1.5$, Grade $4=$ hypereosinophilic syndrome

The aetiology of "abnormal" haematological parameters in African populations remains largely unknown. In our study, males had statistically significantly higher WBC and basophil counts than females (notably, the sample size for females was much smaller than the male sample size). However, we did not find significant associations between haematological parameters and other selected demographic and anthropometric characteristics of the study participants (ethnicity, age, and BMI); this could have been as a result of our limited sample size, especially the number of females. Also, the BMI and age ranges in our study were narrow, and these minimal anthropometric and demographic differences likely contributed greatly to the minimal haematologic differences found among study participants. Most of the participants had normal BMIs $\left(20-25 \mathrm{~kg} / \mathrm{m}^{2}\right)$ and were fairly young adults (age range 19-35 years). Furthermore, we did not collect data on dietary and environmental factors that could affect the haematological parameters, and we did not repeat the tests to ensure accuracy. In general, the main limitation of our study was the small sample size. The targeted sample size of 60 males and 60 females was not reached. Even if these quotas were achieved, they only would have represented $50 \%$ of the recommended sample size and this likely resulted some loss of validity of the study results. Nevertheless, this study works as a proof-of-concept study that can be used to design a more detailed study involving a wide range of age and ethnic subpopulations in order to establish Malawian reference ranges.

Previously, some investigators have suggested that the eosinophilia seen in healthy African subjects results from helminthic infection while anaemia could be because of poor dietary intake of micronutrients, such as iron and folic acid. ${ }^{23}$ Research indicates that total WBC and neutrophil counts are lower among individuals of African descent because of the common African-derived "null" variant (rs2814778) of the Duffy antigen receptor for chemokines (DARC) gene. ${ }^{24-26} \mathrm{It}$ has been shown that by stopping the expression of $D A R C$ on RBCs, the Duffy null variant may alter the concentration and distribution of chemokines in the blood and tissue, ${ }^{27-30}$ thus regulating neutrophil production and migration. This could be another reason for the low WBC counts among the apparently normal Malawian blood donors. This might confirm that some of the apparent haematological abnormalities could simply result from normal genetic variations across different populations. ${ }^{3,5}$ The combination of factors that may affect haematological outcomes may vary by location; studies are, therefore, needed to identify key factors affecting haematological outcomes and to generate specific local haematological reference ranges in different countries and regions. These outcomes highlight the need for a more detailed and robust local study to determine reference ranges for the various haematological variables.

Despite the limitations of this study, some important measures, including those overlooked in other African studies, ${ }^{8,17}$ were seriously taken into consideration. Firstly, our study had a fair representation of the Malawian population because of our eligibility criteria, which excluded subjects with potential risk factors for abnormal haematologic parameters. Secondly, the haematological assays were performed at an internationally reputable laboratory with a good level of adherence to Clinical and Laboratory Standards Institute (CLSI) and Good Clinical Laboratory Practice (GCLP) standards. Thus, our findings are likely to be applicable to a healthy population of Blantyre.

Findings of this study suggest the need to conduct quality studies establishing local reference ranges for haematological variables in countries like Malawi. Such studies should collect data on key behavioural, dietary, and environmental factors that may affect haematological variables. In addition, the studies should prospectively monitor individuals with abnormal results to determine any abnormal clinical outcomes associated with the laboratory abnormalities. Furthermore, it would be necessary to conduct robust immunological tests for individuals with abnormal cell subset white cell counts, so as to establish their cause. Besides this, in clinical practice, abnormal haematological results should be repeated and reconciled with the patient's clinical condition to avoid unnecessary and expensive treatment of otherwise healthy individuals. As is the case with our results; if such "abnormal" results were detected in a clinical setting, a person who does not need treatment would have been treated because of the use of inappropriate reference values. We recommend that future studies should follow-up patients with mild and moderate abnormalities in order to assess potential short- and long-term clinical consequences of the abnormalities. It would be necessary, as well, to use two different analyzers for testing and to perform reproducibility studies on each analyzer. It would also be important to use statistical methodologies that could ensure that the reference ranges generated would clearly distinguish pathology from normal variation, while being a true reflection of the normal Malawian. For instance, the skewed distribution of our data may imply the inclusion of participants with subclinical pathologies. Future studies should conduct additional tests, such as for markers of inflammation (C-reactive protein, for example), to detect underlying pathologies.

In conclusion, data from this study showed that values of haematological variables for a significant number of otherwise normal Malawians were outside a standard haematological machine's reference limits, thereby suggesting abnormalities. This finding is consistent with results from other studies done in some African countries. 


\section{References}

1. Jaoko W, Nakwagala FN, Anzala O, Manyonyi GO, Birungi J, Nanvubya A, et al. Safety and immunogenicity of recombinant lowdosage HIV-1 A vaccine candidates vectored by plasmid pTHr DNA or modified vaccine virus Ankara (MVA) in humans in East Africa. Vaccine. 2008 May 23;26(22):2788-95. doi: 10.1016/j.vaccine.2008.02.071.

2. Kibaya RS, Bautista CT, Sawe FK, Shaffer DN, Sateren WB, Scott PT, et al. Reference ranges for the clinical laboratory derived from a rural population in Kericho, Kenya. PLoS One. 2008 Oct 3;3(10):e3327. doi: 10.1371/journal.pone.0003327.

3. Gill GV, England A, Marshal C. Low platelet counts in Zambians. Trans R Soc Trop Med Hyg. 1979;73(1):111-2.

4. Abdulkadir J, Bolodia G. Haemoglobin and hematocrit levels in young adult Ethiopian males in Addis Ababa. Ethiop Med J. 1979 Jan; 17(1):5-8.

5. Azikiwe AN. Platelet count values in healthy Nigeria medical students in Jos. East Afr Med J. East Afr Med J. 1984 Jun;61(6):482-5.

6. Armstrong P. Full blood count values in adolescents. Ir Med J. 1989 Jun;82(2):68-9.

7. Dal Colletto GM, Fulker DW, Barretto OC, Kolya M. Genetic and environmental effects on blood cells. Acta Genet Med Gemellol (Roma). 1993;42(3-4):245-52.

8. Saathoff E, Schneider P, Kleinfeldt V, Geis S, Haule D, et al. (2008) Laboratory reference values for healthy adults from southern Tanzania. Trop Med Int Health 13: 612-625.

9. Bain BJ. Ethnic and sex differences in the total and differential white cell count and platelet count. J Clin Pathol. 1996 Aug;49(8):664-6.

10. Saxena S, Wong ET. Heterogeneity of common hematologic parameters among racial, ethnic, and gender subgroups. Arch Pathol Lab Med. 1990 Jul;114(7):715-9.

11. Nduka N, Aneka C, Maxwell-Owhochuku S. Comparison of some haematological indices of Africans and Caucasians resident in the same Nigerian environment. Haematologia (Budap). 1988;21(1):57-63.

12. Hawkins WW, Speck E, Leonard VG. Variation of the hemoglobin level with age and sex. Blood. 1954 Oct;9(10):999-1007.

13. Tsegaye A, Messele T, Tilahun T, Hailu E, Sahlu T, Doorly R, et al. Immunohematological reference ranges for adult Ethiopians. Clin Diagn Lab Immunol. 1999 May;6(3):410-4.

14. Lugada ES, Mermin J, Kaharuza F, Ulvestad E, Were W, Langeland $\mathrm{N}$, et al. Population-based hematologic and immunologic reference values for a healthy Ugandan population. Clin Diagn Lab Immunol. 2004 Jan;11(1):29-34.

15. Romeo J, Warnberg J, Gomez-Martinez S, Diaz LE, Moreno LA, Castillo-Garzon M, et al. Hematological reference values in Spanish adolescents: the AVENA study. Eur J Haematol. 2009;83:58-94.

16. Amornkul PN, Vandenhoudt H, Nasokho P, Odhiambo F, Mwaengo $\mathrm{D}$, Hightower A, et al. HIV prevalence and associated risk factors among individuals aged 13-34years in Rural Western Kenya. PLoS One. 2009 Jul 31;4(7):e6470. doi: 10.1371/journal.pone.0006470.

17. Zeh C, Amornkul PN, Inzaule S, Ondoa P, Oyaro B, Mwaengo DM, et al. Population-based biochemistry, immunologic and hematological reference values for adolescents and young adults in a rural population in Western Kenya. PLoS One. 2011;6(6):e21040. doi: 10.1371/journal. pone.0021040.

18. El-Hazmi MA, Al-Faleh FZ, Al-Mofleh IA, Warsy AS, Al-Askah AK. Establishment of normal "reference" ranges for hematological parameters for healthy Saudi Arabs. Trop Geogr Med. 1982;34(4):3339.
19. NCCLS (2000) How to define and determine reference intervals in the clinical laboratory; approved guideline-Second Edition. National Committee for Clinical Laboratory Standards, Wayne, PA C28-A2, Vol. 20 No 13. 2ed.

20. National Institute of Allergy and Infectious Diseases. Division of AIDS (DAIDS) table for grading the severity of adult and pediatric adverse events [Internet]. Bethesda, MD, USA: National Institute of Allergy and Infectious Diseases; 2004 [cited 2015 Mar 30]. Available from: http://www.niaid.nih.gov/labsandresources/resources/ daidsclinrsrch/documents/daidsaegradingtable.pdf.

21. Owusu-Agyei S, Ansong D, Asante K, Kwarteng Owusu S, Owusu R, Wireko Brobby NA, et al. Randomized controlled trial of RTS,S/ AS02D and RTS,S/AS01E malaria candidate vaccines given according to different schedules in Ghanaian children. PLoS One. 2009 Oct 2;4(10):e7302. doi: 10.1371/journal.pone.0007302.

22. Badenhorst CJ, Fourie J, Steyn K, Jooste PL, Lombard CJ, Bourne $\mathrm{L}$, et al. The haematological profile of urban black Africans aged 15-64 years in the Cape Peninsula. East Afr Med J. 1995 Jan;72(1):19-24.

23. Mathanga DP, Campbell CH Jr, Vanden Eng J, Wolkon A, Bronzan $\mathrm{RN}$, Malenga GJ, et al. Comparison of anaemia and parasitaemia as indicators of malaria control in household and EPI-health facility surveys in Malawi. Malar J. 2010 Apr 21;9:107. doi: 10.1186/14752875-9-107.

24. Nalls MA, Wilson JG, Patterson NJ, Tandon A, Zmuda JM, Huntsman S, et al. Admixture mapping of white cell count: genetic locus responsible for lower white blood cell count in the Health $\mathrm{ABC}$ and Jackson Heart studies. Am J Hum Genet. 2008 Jan;82(1):81-7. doi: 10.1016/j.ajhg.2007.09.003.

25. Reich D, Nalls MA, Kao WH, Akylbekova EL, Tandon A, Patterson $\mathrm{N}$, et al. Reduced neutrophil count in people of African descent is due to a regulatory variant in the Duffy antigen receptor for chemokines gene. PLoS Genet. 2009 Jan;5(1):e1000360. doi: 10.1371/journal. pgen. 1000360 .

26. Lo KS, Wilson JG, Lange LA, Folsom AR, Galarneau G, Ganesh SK, et al. Genetic association analysis highlights new loci that modulate hematological trait variation in Caucasians and African Americans. Hum Genet. 2011 Mar;129(3):307-17. doi: 10.1007/s00439-010-09251 .

27. Dawson TC, Lentsch AB, Wang Z, Cowhig JE, Rot A, Maeda N, et al. Exaggerated response to endotoxin in mice lacking the Duffy antigen/ receptor for chemokines (DARC). Blood. 2000 Sep 1;96(5):1681-4.

28. Lee JS, Wurfel MM, Matute-Bello G, Frevert CW, Rosengart MR, Ranganathan M, et al. The Duffy antigen modifies systemic and local tissue chemokine responses following lipopolysaccharide stimulation. J Immunol. 2006 Dec 1;177(11):8086-94.

29. Reutershan J, Harry B, Chang D, Bagby GJ, Ley K. DARC on RBC limits lung injury by balancing compartmental distribution of CXC chemokines. Eur J Immunol. 2009 Jun;39(6):1597-607. doi: 10.1002/ eji.200839089.

30. Schnabel RB, Baumert J, Barbalic M, Dupuis J, Ellinor PT, Durda P, et al. (2010) Duffy antigen receptor for chemokines (Darc) polymorphism regulates circulating concentrations of monocyte chemoattractant protein-1 and other inflammatory mediators. Blood. 2010 Jul 1;115(26):5289-99. doi: 10.1182/blood-2009-05-221382. 